\title{
A construction for biembeddings of Latin squares
}

\author{
M. J. Grannell \\ Department of Mathematics and Statistics \\ The Open University \\ Walton Hall \\ Milton Keynes MK7 6AA \\ United Kingdom \\ m.j.grannell@open.ac.uk
}

\author{
M. Knor \\ Department of Mathematics \\ Faculty of Civil Engineering \\ Slovak University of Technology \\ Radlinského 11 \\ 81368 Bratislava \\ Slovakia \\ knor@math.sk
}

Submitted: Dec 13, 2010; Accepted: Sep 14, 2011; Published: Sep 26, 2011

Mathematics Subject Classifications: 05B15, 05C10

\begin{abstract}
An existing construction for face 2-colourable triangular embeddings of complete regular tripartite graphs is extended and then re-examined from the viewpoint of the underlying Latin squares. We prove that this generalization gives embeddings which are not isomorphic to any of those produced by the original construction.
\end{abstract}

\section{Introduction}

Establishing the existence of a minimum genus surface embedding of each complete graph $K_{n}$ was a crucial step in Ringel and Youngs' solution of the famous Heawood map colouring problem for surfaces of positive genus [17]. For some residue classes modulo 12 such embeddings necessarily have all their faces triangular. Until 1999 the maximum number of known nonisomorphic triangular embeddings of $K_{n}$ for any particular $n$ in either an orientable or nonorientable surface was a mere three [16]. Subsequent papers have established that the number of such embeddings grows exponentially with $n[1,8,11,12,13,14,15]$. This number cannot exceed $n^{n^{2} / 3}$ and the best known lower bound, for a restricted infinite class of values of $n$, is of the form $n^{a n^{2}}$, where $a$ is just less than $1 / 864[4,7,9]$. A major component of the proof of this best known lower bound is a recursive construction given in [8] for face 2-colourable triangular embeddings of the complete tripartite graph $K_{n, n, n}$. There is something of a lack of general constructions for such embeddings so extensions of existing constructions are of interest. In the current paper we describe a generalization of this construction that removes the need for a parallel class in one of the colours. We 
also identify the corresponding Latin squares, thereby generalizing the main result of [7]. A rather restricted version of this generalization was given in [3]. We prove that the fuller version given here generates new embeddings which are not obtainable from the earlier constructions given in $[3,7,8]$.

The surfaces we consider will be closed, connected 2-manifolds, without a boundary. Given a triangular embedding of some simple graph $G$ with vertex set $V(G)$, the rotation at a vertex $v \in V(G)$ is the cyclically ordered permutation of vertices adjacent to $v$, with the ordering determined by the embedding. Conversely, given a set of triangular faces with each edge appearing in precisely two faces, the faces may be sewn together along their common edges. If at each vertex $v$ the resulting permutation of neighbouring vertices is a single cycle, then the faces form a triangulation of some surface with the cycle at $v$ forming the rotation at $v$.

If row $i$, column $j$ contains entry $k$ in the Latin square $L$ then we write $k=L(i, j)$ or $(i, j, k) \in L$. When a triangular embedding of $K_{n, n, n}$ is face 2-colourable, the triangular faces in each colour class determine a Latin square of order $n$ by taking these faces as the (row, column, entry) triples, where the row labels, the column labels and the entries form the three sets of the partition. Thus a face 2-colourable triangular embedding of $K_{n, n, n}$ may alternatively be described as a biembedding of the associated Latin squares and we may use the terms triangles, triangular faces or triples interchangeably to refer to the faces of the embeddings or to the triples of the Latin squares. The colour classes of a face 2-coloured embedding will be taken as black and white. We write $A \bowtie B$ to denote the fact that the Latin square $A$ biembeds with the Latin square $B$, and we also use this notation to denote the biembedding itself, taking $A$ white and $B$ black. It was shown in [5], by a very easy argument, that a face 2-colourable triangular embedding of $K_{n, n, n}$, i.e. a biembedding of two Latin squares, is necessarily in an orientable surface.

A parallel class in a triangular embedding of $K_{n, n, n}$ is a set of $n$ triangular faces that cover all $3 n$ vertices. A parallel class in one colour class of a face 2-colourable triangular embedding of $K_{n, n, n}$ corresponds to a transversal in the associated Latin square of side $n$, that is to say a set of $n$ cells from the square that contains every entry symbol, and covers every row and every column. A parallel class containing triangular faces from both colour classes corresponds to a shared transversal having some cells from each of the two Latin squares. Formally, $\left(\mathcal{T}_{1}, \mathcal{T}_{2}\right)$ is a shared transversal of a biembedding $L_{1} \bowtie L_{2}$ of two Latin squares of side $n$ if $\mathcal{T}_{1}$ is a partial transversal of $L_{1}, \mathcal{T}_{2}$ is a partial transversal of $L_{2}$, $\left|\mathcal{T}_{1} \cup \mathcal{T}_{2}\right|=n$, and the triples of $\mathcal{T}_{1} \cup \mathcal{T}_{2}$ cover every row, every column and every entry symbol.

We say that the Latin square $B$ is isotopic to $A$ if $A$ can be transformed to $B$ by applying permutations $\phi_{r}, \phi_{c}$ and $\phi_{e}$ respectively to the row labels, column labels, and entries. We say that two embeddings $\mu$ and $\nu$ of the same graph $G$ are isomorphic if there exists a permutation of the vertices of $G$ that maps edges of $\mu$ to edges of $\nu$ and faces of $\mu$ to faces of $\nu$.

For any remaining undefined items of terminology and for background information we refer the reader to $[2,10,17]$.

In the next section we describe a generalization of the recursive construction given in 
[8] for face 2-colourable triangular embeddings of complete bipartite graphs $K_{n, n, n}$. The original construction can be viewed in terms of the underlying Latin squares as described in the following theorem taken from [7].

Theorem 1.1 Suppose that, for $0 \leq u \leq m-1, L \bowtie L_{u}^{\prime}$, where $L$ and each $L_{u}^{\prime}$ are of order $n$ and have row, column and entry labels $\{0,1, \ldots, n-1\}$. Suppose also that for each $(i, j)$ with $0 \leq i, j \leq n-1, Q_{i, j} \bowtie Q_{i, j}^{\prime}$, where both $Q_{i, j}$ and $Q_{i, j}^{\prime}$ are of order $m$, and have row, column and entry labels $\{0,1, \ldots, m-1\}$, and that the squares $Q_{i, j}^{\prime}$ have a transversal $\mathcal{T}$ which is common for all pairs $(i, j)$. Define $A$ and $A^{\prime}$, Latin squares of order $m n$ with row, column and entry labels $\{0,1, \ldots, m n-1\}$, by

$$
\begin{gathered}
A(n u+i, n v+j)=n Q_{i, j}(u, v)+L(i, j), \\
A^{\prime}(n u+i, n v+j)=n Q_{i, j}^{\prime}(u, v)+k^{\prime}
\end{gathered}
$$

for $0 \leq u, v \leq m-1$ and $0 \leq i, j \leq n-1$. Then $A \bowtie A^{\prime}$.

Both the original construction and the version of it given in Theorem 1.1 require the existence of a transversal in one colour class of some of the constituent Latin squares. Our generalization, presented in Section 2, permits the transversal to be shared between the two colour classes; this considerably extends the applicability of the construction. In Section 3 we present an infinite class of biembeddings, none of which can be obtained from Theorem 1.1 with $m, n>1$ for any choices of the $m$ biembeddings $L \bowtie L_{u}^{\prime}$ and $n^{2}$ biembeddings $Q_{i, j} \bowtie Q_{i, j}^{\prime}$. Furthermore, each of these new biembeddings is constructed in the simplest possible way by our generalized construction, using just two biembeddings $L \bowtie L^{\prime}$ and $Q \bowtie Q^{\prime}$.

\section{Construction}

The original construction from [8] produces a face 2-colourable triangular embedding of $K_{m n, m n, m n}$ from face 2-colourable triangular embeddings of $K_{m, m, m}$ and $K_{n, n, n}$. We now describe our generalization using a labelling of the vertices of the embeddings that will help in the subsequent discussion when we relate the construction to Latin squares.

So, suppose that for $0 \leq u \leq m-1, \phi_{u}$ is a face 2-coloured triangular embedding of $K_{n, n, n}$ with vertex set $R_{u} \cup C_{u} \cup E_{u}$, where $R_{u}=\left\{r_{n u+i}: 0 \leq i \leq n-1\right\}, C_{u}=$ $\left\{c_{n u+i}: 0 \leq i \leq n-1\right\}$ and $E_{u}=\left\{e_{n u+i}: 0 \leq i \leq n-1\right\}$ are three disjoint sets. We use the letters $r, c$ and $e$ because these will later be related to the row, column and entry labels of a Latin square. We also suppose that for each oriented white triangle $\left(r_{i}, c_{j}, e_{k}\right)$ $\left(=\left(r_{n 0+i}, c_{n 0+j}, e_{n 0+k}\right)\right)$ of $\phi_{0}$, the ordered triple $\left(r_{n u+i}, c_{n u+j}, e_{n u+k}\right)$ defines an oriented white triangle of $\phi_{u}$ for each $u \in\{0,1, \ldots, m-1\}$. In essence, this means that the embeddings $\phi_{u}$ all have the "same" white triangles with the same orientations, although the black triangles may be different. Then, for each pair $(i, j)$ with $0 \leq i, j \leq n-1$, 
suppose that $\psi_{i, j}$ is a face 2-coloured triangular embedding of $K_{m, m, m}$, and that all of these embeddings have a common parallel class $\mathcal{P}$ of triangular faces of which $m_{1}$ are black and $m_{2}$ are white, so that $m_{1}+m_{2}=m$. (The construction in [3] dealt with the special case when $m=2$ and $m_{1}=m_{2}=1$.) Initially, the supporting surfaces of all the embeddings $\phi_{u}$ and $\psi_{i, j}$ are taken to be disjoint from one another. For each $u$, let $\phi_{u}^{\prime}$ be the embedding obtained from $\phi_{u}$ by reversing the colours and the orientation.

Next list the $n^{2}$ white triangles of $\phi_{0}$; without loss of generality, we can take these as given by $W_{i, j}=\left(r_{i}, c_{j}, e_{k}\right)$, where $k$ is uniquely determined by $(i, j)$. Then label the $m$ triangles forming the parallel class $\mathcal{P}$ in $\psi_{i, j}$ as $\left(r_{n u+i}^{*}, c_{n u+j}^{*}, e_{n u+k}^{*}\right), 0 \leq u \leq m-1$, taking care to respect the three vertex parts of the embedding, so that one vertex part receives labels $r^{*}$, another $c^{*}$ and the third $e^{*}$. The order in which the triangles of $\mathcal{P}$ are taken is immaterial except that the colours should be consistent, so that if $\left(r_{n u+i}^{*}, c_{n u+j}^{*}, e_{n u+k}^{*}\right)$ is black (respectively, white) then $\left(r_{n u+i^{\prime}}^{*}, c_{n u+j^{\prime}}^{*}, e_{n u+k^{\prime}}^{*}\right)$ is black (white) for all $\left(i^{\prime}, j^{\prime}\right)$. Then each vertex of $\psi_{i, j}$ is uniquely labelled. For each black triangle $\left(r_{n u+i}^{*}, c_{n u+j}^{*}, e_{n u+k}^{*}\right)$ of $\mathcal{P}$ there is a corresponding oriented white triangle $\left(r_{n u+i}, c_{n u+j}, e_{n u+k}\right)$ of the embedding $\phi_{u}$, and for each white triangle $\left(r_{n u+i}^{*}, c_{n u+j}^{*}, e_{n u+k}^{*}\right)$ of $\mathcal{P}$ there is a corresponding oriented black triangle $\left(r_{n u+i}, c_{n u+j}, e_{n u+k}\right)$ of the embedding $\phi_{u}^{\prime}$. We take the orientation of $\psi_{i, j}$ to be such that these triangles are oriented oppositely to the corresponding ones of $\phi_{u}$ and $\phi_{u}^{\prime}$. Figure 1 illustrates the situation in the particular case when the first $m_{1}$ triangles of $\mathcal{P}$ are black and the last $m_{2}$ triangles of $\mathcal{P}$ are white.

Finally, cut out from the supporting surfaces each such pair of corresponding triangles, one pair at a time, and identify the corresponding vertices $\left(x^{*}\right.$ with $\left.x\right)$ and edges bordering the two holes. After dealing with all $m n^{2}$ pairs of corresponding triangles in this fashion the result is a face 2-colourable triangular embedding $\chi$ of some graph $G$. To see that $G=K_{m n, m n, m n}$, note that the vertex set of $G$ is $\bar{R} \cup \bar{C} \cup \bar{E}$, where $\bar{R}=\left\{r_{n u+i}: 0 \leq\right.$ $u \leq m-1,0 \leq i \leq n-1\}, \bar{C}=\left\{c_{n u+i}: 0 \leq u \leq m-1,0 \leq i \leq n-1\right\}$ and $\bar{E}=\left\{e_{n u+i}: 0 \leq u \leq m-1,0 \leq i \leq n-1\right\}$ are three disjoint sets. No edges are present in $G$ between any two vertices in $\bar{R}$, and likewise for $\bar{C}$ and $\bar{E}$. Every edge $r c$ with $r \in \bar{R}$ and $c \in \bar{C}$ appears precisely once in $G$, and likewise for the pairs $\bar{C} \bar{E}$ and $\bar{E} \bar{R}$. For example, the edge $r_{n u+i} c_{n u+j}$ is only in $\phi_{u}$ and $\psi_{i, j}$. After cutting out the triangles, this edge borders a hole in both embeddings, so that after gluing it appears exactly once in $\chi$. On the other hand the edge $r_{n u+i} c_{n v+j}, u \neq v$, is only in $\psi_{i, j}$, and consequently it too appears exactly once in $\chi$.

We are now in a position to state our main result which interprets the above construction in terms of Latin squares. Using relabellings, there are many pairs of Latin squares that can be used to represent the embedding $\chi$. Theorem 2.1 gives, what appears to us, the most useful representation. 


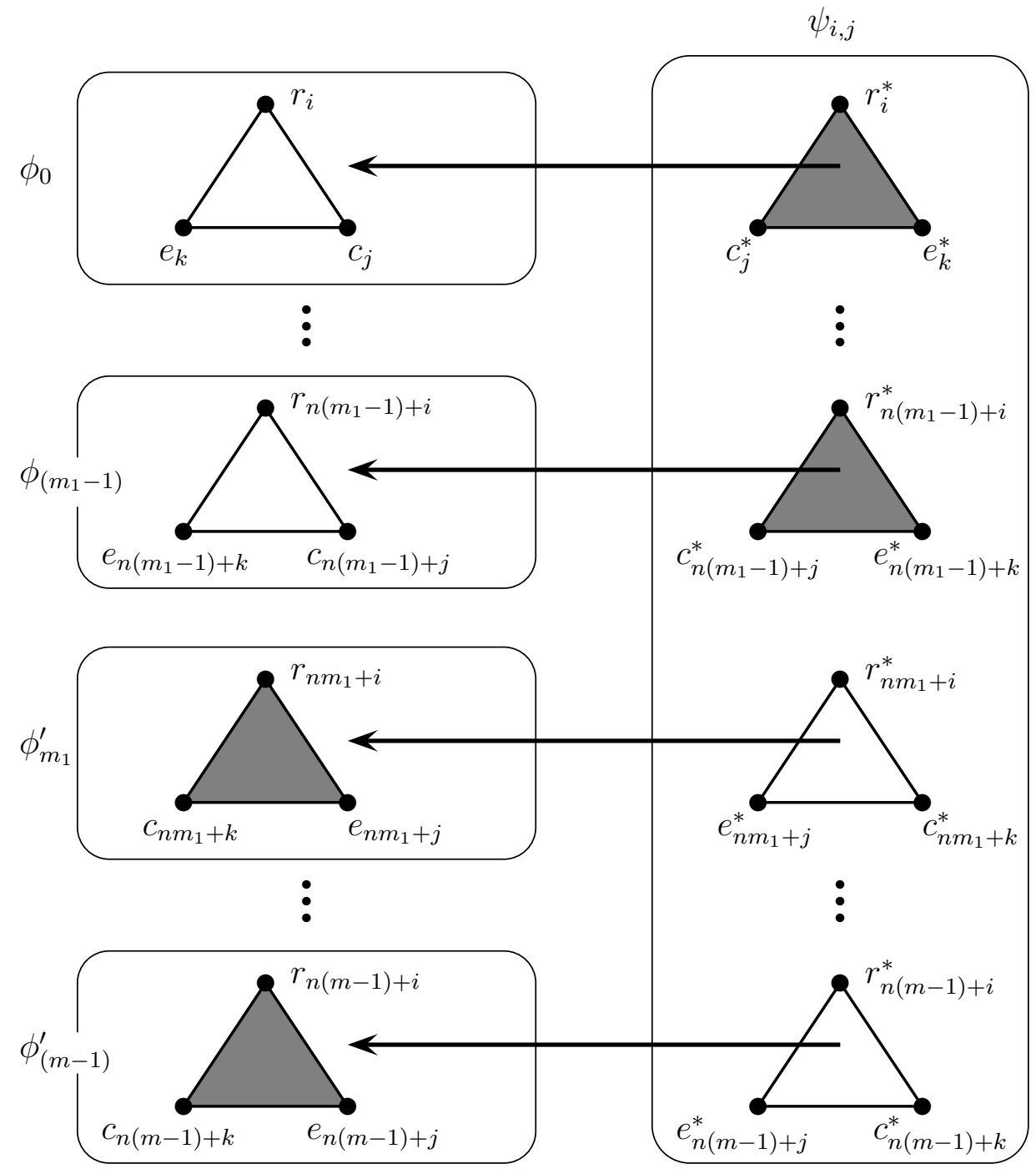

Each $\phi_{u}$ is an embedding of $K_{n, n, n}$ and $\psi_{i, j}$ is an embedding of $K_{m, m, m}$.

Figure 1. The construction.

Theorem 2.1 Suppose that, for $0 \leq u \leq m-1, L \bowtie L_{u}^{\prime}$, where $L$ and each $L_{u}^{\prime}$ are of order $n$ and have row, column and entry labels $\{0,1, \ldots, n-1\}$. Suppose also that for each $(i, j)$ with $0 \leq i, j \leq n-1, Q_{i, j} \bowtie Q_{i, j}^{\prime}$, where both $Q_{i, j}$ and $Q_{i, j}^{\prime}$ are of order $m$, and have row, column and entry labels $\{0,1, \ldots, m-1\}$, and that the squares $Q_{i, j}$ and $Q_{i, j}^{\prime}$ have a shared transversal $\mathcal{T}$ which is common for all pairs $(i, j)$. Define $A$ and $A^{\prime}$, Latin squares of order $m n$ with row, column and entry labels $\{0,1, \ldots, m n-1\}$, by

$$
\begin{aligned}
A(n u+i, n v+j) & =n Q_{i, j}(u, v)+k \\
A^{\prime}(n u+i, n v+j) & =n Q_{i, j}^{\prime}(u, v)+k^{\prime}
\end{aligned}
$$




$$
\begin{aligned}
& \text { where } k= \begin{cases}L_{u}^{\prime}(i, j) & \text { if there exists } w \text { such that }(u, v, w) \in \mathcal{T} \cap Q_{i, j}, \\
L(i, j) & \text { otherwise, }\end{cases} \\
& \text { and } k^{\prime}= \begin{cases}L_{u}^{\prime}(i, j) & \text { if there exists } w \text { such that }(u, v, w) \in \mathcal{T} \cap Q_{i, j}^{\prime}, \\
L(i, j) & \text { otherwise, }\end{cases}
\end{aligned}
$$

for $0 \leq u, v \leq m-1$ and $0 \leq i, j \leq n-1$. Then $A \bowtie A^{\prime}$.

Proof. Our proof makes use of the terminology established in the preceeding discussion, which is similar to the terminology employed in [7]. Using $\phi_{u}=L \bowtie L_{u}^{\prime}$ and $\psi_{i, j}=Q_{i, j} \bowtie$ $Q_{i, j}^{\prime}$, the embedding $\chi$ of $K_{m n, m n, m n}$ is produced as described above. The Latin squares $S$ and $S^{\prime}$ such that $\chi=S \bowtie S^{\prime}$ are then identified. Finally, permutations of the columns and entries of $S$ and $S^{\prime}$ give $A$ and $A^{\prime}$, thereby establishing that $A \bowtie A^{\prime}$.

Throughout the proof and subsequent discussions, we take the triangles determined by $Q_{i, j}$ and $A$ to be white, and those determined by $Q_{i, j}^{\prime}$ and $A^{\prime}$ to be black. Note that the biembeddings $L \bowtie L_{u}^{\prime}$ all have a common set of triangles determined by $L$. For each $u$, we relabel the two Latin squares of the biembedding $L \bowtie L_{u}^{\prime}$ by adding $n u$ to each of the row, column and entry labels. This converts them to the range $\{n u, n u+$ $1, n u+2, \ldots, n u+n-1\}$. The resulting $m$ embeddings of $K_{n, n, n}$ may then be represented on $m$ disjoint surfaces, and the vertex set of the $u^{\text {th }}$ embedding $\phi_{u}$ may be taken as $R_{u} \cup C_{u} \cup E_{u}$, where $R_{u}=\left\{r_{n u+i}: 0 \leq i \leq n-1\right\}, C_{u}=\left\{c_{n u+i}: 0 \leq i \leq n-1\right\}$ and $E_{u}=\left\{e_{n u+i}: 0 \leq i \leq n-1\right\}$ are three disjoint sets representing the rows, columns and entries of the relabelled Latin squares. The triple $\left(r_{n u+i}, c_{n u+j}, e_{n u+k}\right)$ will be taken as a white triangle of $\phi_{u}$ if and only if $k=L(i, j)$, and as a black triangle of $\phi_{u}$ if and only if $k=L_{u}^{\prime}(i, j)$. The embedding $\phi_{u}^{\prime}$ is obtained by reversing both the orientation and the colouring of $\phi_{u}$.

Next suppose that $\mathcal{T}=\left\{\left(\alpha_{u}, \beta_{u}, \gamma_{u}\right): 0 \leq u \leq m-1\right\}$ where, for each $u, \gamma_{u}=$ $Q_{i, j}^{\prime}\left(\alpha_{u}, \beta_{u}\right)$ or $Q_{i, j}\left(\alpha_{u}, \beta_{u}\right)$ (according as to whether the triple lies in $Q_{i, j}^{\prime}$ or $Q_{i, j}$ ) for every $(i, j)$. Note that $\left\{\alpha_{u}: 0 \leq u \leq m-1\right\}=\{0,1, \ldots, m-1\}$, and similarly for $\beta$ and $\gamma$. Also, without loss of generality, we may take $\alpha_{u}=u$ for each $u$. For each of the $n^{2}$ pairs $(i, j)$, we relabel the two Latin squares $Q_{i, j}$ and $Q_{i, j}^{\prime}$ using the triangle $(i, j, k)$ defined by $k=L(i, j)$ and the transversal $\mathcal{T}$; each row label $\alpha_{u}$ is renamed as $n u+i$, each column label $\beta_{u}$ is renamed as $n u+j$, and each entry label $\gamma_{u}$ is renamed as $n u+k$. Since $\mathcal{T}$ covers all row labels, column labels and entry labels of $Q_{i, j}$ and $Q_{i, j}^{\prime}$, each of these labels is renamed by this process. The resulting $n^{2}$ embeddings of $K_{m, m, m}$ may then be represented on $n^{2}$ disjoint surfaces, which we will take to be disjoint from those of the biembeddings $\phi_{u}$. The vertex set of the $(i, j)^{\text {th }}$ embedding $\psi_{i, j}$ may be taken as $R_{i, j}^{*} \cup C_{i, j}^{*} \cup E_{i, j}^{*}$, where $R_{i, j}^{*}=\left\{r_{n u+i}^{*}: 0 \leq u \leq m-1\right\}, C_{i, j}^{*}=\left\{c_{n u+j}^{*}: 0 \leq u \leq m-1\right\}$ and $E_{i, j}^{*}=\left\{e_{n u+k}^{*}: 0 \leq u \leq m-1\right\}$ are three disjoint sets representing the rows, columns and entries of the relabelled Latin square.

Thus the triple $\left(r_{n u+i}^{*}, c_{n v+j}^{*}, e_{n w+k}^{*}\right)$ defines a white triangle of $\psi_{i, j}$ if and only if $k=$ $L(i, j)$ and $\gamma_{w}=Q_{i, j}\left(\alpha_{u}, \beta_{v}\right)$, and it defines a black triangle of $\psi_{i, j}$ if and only if $k=L(i, j)$ and $\gamma_{w}=Q_{i, j}^{\prime}\left(\alpha_{u}, \beta_{v}\right)$. Note that if $k=L(i, j)$ then $\left(r_{n u+i}^{*}, c_{n u+j}^{*}, e_{n u+k}^{*}\right)$ is a black or white triangle of $\psi_{i, j}$ according as to whether $\left(\alpha_{u}, \beta_{u}, \gamma_{u}\right)$ is a black or white triple of $\mathcal{T}$, that is to say whether it lies in $\mathcal{T} \cap Q_{i, j}^{\prime}$ or in $\mathcal{T} \cap Q_{i, j}$. 
With the relabellings described in the previous two paragraphs, the biembeddings $\phi_{l}, \phi_{l}^{\prime}$ and $\psi_{i, j}$ correspond precisely to the construction described at the start of this section. By cutting out from the supporting surfaces each pair of corresponding triangles, and identifying the corresponding vertices and edges, the result is a face 2-colourable triangular embedding $\chi$ of $K_{m n, m n, m n}$. Our labelling of the points gives this embedding on the vertex set $\bar{R} \cup \bar{C} \cup \bar{E}$, where $\bar{R}=\left\{r_{n u+i}: 0 \leq u \leq m-1,0 \leq i \leq n-1\right\}$, $\bar{C}=\left\{c_{n u+i}: 0 \leq u \leq m-1,0 \leq i \leq n-1\right\}$ and $\bar{E}=\left\{e_{n u+i}: 0 \leq u \leq m-1,0 \leq i \leq n-1\right\}$ are three disjoint sets. We next identify for $\chi$ the two Latin squares $S$ (white) and $S^{\prime}$ (black) for which the biembedding $S \bowtie S^{\prime}$ gives $\chi$.

Take first a typical white triangle of $\chi$ having the edge $\left\{r_{n u+i}, c_{n v+j}\right\}$. If $u \neq v$, or if $u=v$ and $\left(\alpha_{u}, \beta_{u}, \gamma_{u}\right)$ is a triple from $Q_{i, j}^{\prime}$ (i.e. if $\left(\alpha_{u}, \beta_{u}, \gamma_{u}\right) \in \mathcal{T}$ is black), then this triangle comes from the embedding $\psi_{i, j}$, and so the third vertex is $e_{n w+k}$ where $k=L(i, j)$ and $w$ is given by $\gamma_{w}=Q_{i, j}\left(\alpha_{u}, \beta_{v}\right)$. But if $u=v$ and $\left(\alpha_{u}, \beta_{u}, \gamma_{u}\right)$ is a triple from $Q_{i, j}$, then this triangle comes from the embedding $\phi_{u}^{\prime}$ (where the colours of $\phi_{u}$ are reversed), and so the third vertex is $e_{n u+k}$ where $k=L_{u}^{\prime}(i, j)$. Thus the Latin square $S$ giving the white triangles of $\chi$ is represented with row, column and entry labels $\{0,1, \ldots, m n-1\}$ by

$$
\begin{aligned}
S(n u+i, n v+j) & =n w+k, \\
\text { where } \gamma_{w} & =Q_{i, j}\left(\alpha_{u}, \beta_{v}\right) \\
\text { and } k & =\left\{\begin{array}{l}
L_{u}^{\prime}(i, j) \text { if } u=v \text { and }\left(\alpha_{u}, \beta_{u}, \gamma_{u}\right) \in Q_{i, j}, \\
L(i, j) \text { otherwise. }
\end{array}\right.
\end{aligned}
$$

Take next a typical black triangle of $\chi$ having the edge $\left\{r_{n u+i}, c_{n v+j}\right\}$. If $u \neq v$, or if $u=v$ and $\left(\alpha_{u}, \beta_{u}, \gamma_{u}\right)$ is a triple from $Q_{i, j}$, then this triangle comes from the embedding $\psi_{i, j}$, and so the third vertex is $e_{n w+k}$ where $k=L(i, j)$ and $w$ is given by $\gamma_{w}=Q_{i, j}^{\prime}\left(\alpha_{u}, \beta_{v}\right)$. But if $u=v$ and $\left(\alpha_{u}, \beta_{u}, \gamma_{u}\right)$ is a triple from $Q_{i, j}^{\prime}$, then this triangle comes from the embedding $\phi_{u}$, and so the third vertex is $e_{n u+k}$ where $k=L_{u}^{\prime}(i, j)$. Thus the Latin square $S^{\prime}$ giving the black triangles of $\chi$ is represented with row, column and entry labels $\{0,1, \ldots, m n-1\}$ by

$$
\begin{aligned}
S^{\prime}(n u+i, n v+j) & =n w+k \\
\text { where } \gamma_{w} & =Q_{i, j}^{\prime}\left(\alpha_{u}, \beta_{v}\right) \\
\text { and } k & =\left\{\begin{array}{l}
L_{u}^{\prime}(i, j) \text { if } u=v \text { and }\left(\alpha_{u}, \beta_{u}, \gamma_{u}\right) \in Q_{i, j}^{\prime}, \\
L(i, j) \text { otherwise. }
\end{array}\right.
\end{aligned}
$$

Our final step is to permute the row, column and entry labels of $S$ and $S^{\prime}$ to give new Latin squares $A$ and $A^{\prime}$. We define

$$
\begin{aligned}
A\left(n \alpha_{u}+i, n \beta_{v}+j\right) & =n \gamma_{w}+k \quad \Leftrightarrow \quad S(n u+i, n v+j)=n w+k \\
A^{\prime}\left(n \alpha_{u}+i, n \beta_{v}+j\right) & =n \gamma_{w}+k \Leftrightarrow S^{\prime}(n u+i, n v+j)=n w+k
\end{aligned}
$$

where $u, v, w \in\{0,1, \ldots, m-1\}$ and $i, j, k \in\{0,1, \ldots, n-1\}$. For example, the column $n v+j$ of $S$ becomes the column $n \beta_{v}+j$ of $A$. In fact, the row labels are unaltered because 
we have taken $\alpha_{u}=u$. Since $S \bowtie S^{\prime}$, we have $A \bowtie A^{\prime}$. Noting that $L_{\alpha_{u}}^{\prime}=L_{u}^{\prime}$, we have for $0 \leq u, v \leq m-1$ and $0 \leq i, j \leq n-1$,

$$
\begin{aligned}
A(n u+i, n v+j) & =n Q_{i, j}(u, v)+k \\
A^{\prime}(n u+i, n v+j) & =n Q_{i, j}^{\prime}(u, v)+k^{\prime}
\end{aligned}
$$

where $k= \begin{cases}L_{u}^{\prime}(i, j) & \text { if there exists } w \text { such that }(u, v, w) \in \mathcal{T} \cap Q_{i, j}, \\ L(i, j) & \text { otherwise, }\end{cases}$

and $k^{\prime}= \begin{cases}L_{u}^{\prime}(i, j) & \text { if there exists } w \text { such that }(u, v, w) \in \mathcal{T} \cap Q_{i, j}^{\prime}, \\ L(i, j) & \text { otherwise. }\end{cases}$

This completes the proof.

Corollary 2.2 below gives a simplified version of Theorem 2.1, obtained by taking $L_{u}^{\prime}$ to be independent of $u$, and $Q_{i, j}$ and $Q_{i, j}^{\prime}$ to be independent of $(i, j)$. It also introduces a notation for the resulting squares $A$ and $A^{\prime}$ to emphasize their dependency on $L, L^{\prime}, Q$ and $Q^{\prime}$. The corollary and the notation will be used in the next section.

Corollary 2.2 Suppose that $L \bowtie L^{\prime}$, where $L$ and $L^{\prime}$ are of order $n$ and have row, column and entry labels $\{0,1, \ldots, n-1\}$. Suppose also that $Q \bowtie Q^{\prime}$, where $Q$ and $Q^{\prime}$ are of order $m$ and have row, column and entry labels $\{0,1, \ldots, m-1\}$, and that the squares $Q$ and $Q^{\prime}$ have a shared transversal $\mathcal{T}$. Define $Q\left(L, \mathcal{T}, L^{\prime}\right)$ and $Q^{\prime}\left(L, \mathcal{T}, L^{\prime}\right)$ to be Latin squares of order mn with row, column and entry labels $\{0,1, \ldots, m n-1\}$, by

$$
\begin{aligned}
Q\left(L, \mathcal{T}, L^{\prime}\right)(n u+i, n v+j) & =n Q(u, v)+k, \\
Q^{\prime}\left(L, \mathcal{T}, L^{\prime}\right)(n u+i, n v+j) & =n Q^{\prime}(u, v)+k^{\prime},
\end{aligned}
$$

where $k= \begin{cases}L^{\prime}(i, j) & \text { if there exists } w \text { such that }(u, v, w) \in \mathcal{T} \cap Q, \\ L(i, j) & \text { otherwise, }\end{cases}$

and $k^{\prime}= \begin{cases}L^{\prime}(i, j) & \text { if there exists } w \text { such that }(u, v, w) \in \mathcal{T} \cap Q^{\prime}, \\ L(i, j) & \text { otherwise, }\end{cases}$

for $0 \leq u, v \leq m-1$ and $0 \leq i, j \leq n-1$. Then $Q\left(L, \mathcal{T}, L^{\prime}\right) \bowtie Q^{\prime}\left(L, \mathcal{T}, L^{\prime}\right)$.

\section{$3 \quad$ New biembeddings}

To demonstrate Theorem 2.1 we present an infinite class of biembeddings of Latin squares, which cannot be obtained by the previous product constructions from $[3,8]$ in a nontrivial way. By "non-trivial" we mean that both factors have orders strictly greater than 1. However, first we present a lemma about the "white" square of Theorem 1.1.

Lemma 3.1 Let $A$ be the Latin square from Theorem 1.1. Then $A$ is decomposable into $n^{2}$ Latin squares $A_{i, j}$ of order $m, 0 \leq i, j \leq m-1$, defined by $A_{i, j}(u, v)=A(n u+i, n v+j), 0 \leq$ $u, v \leq m-1$, with entry labels $\left\{A_{i, j}(u, v) ; 0 \leq u, v \leq m-1\right\}=\{t, n+t, \ldots,(m-1) n+t\}$ for $t=L(i, j)$. 
Proof. Fix $i$ and $j, 0 \leq i, j \leq n-1$, and put $t=L(i, j)$. Consider the square subarray $A_{i, j}(u, v)=A(n u+i, n v+j)$ of order $m, 0 \leq u, v \leq m-1$. Since $A_{i, j}(u, v)=$ $A(n u+i, n v+j)=n Q_{i, j}(u, v)+L(i, j)=n Q_{i, j}(u, v)+t$, the entries of $A_{i, j}$ are elements of the set $\{t, n+t, \ldots,(m-1) n+t\}$. Since there are exactly $m$ of these elements and $m$ is the order of $A_{i, j}$, the sub-array $A_{i, j}$ is a Latin square. Obviously, $\left\{A_{i, j} ; 0 \leq i, j \leq n-1\right\}$ forms a decomposition of $A$.

We remark that the Latin square $A$ from Theorem 1.1 is not necessarily decomposable into Latin squares of order $n$, as can be deduced from Example 2.3 of [7].

By $C_{n}$ we denote the cyclic Latin square of order $n$ formed from the Cayley table of the group $\left(\mathbb{Z}_{n}, \oplus\right)$. That is, $C_{n}(i, j)=i \oplus j$, where the addition is considered modulo $n$. As our application of Theorem 2.1 is based on cyclic Latin squares, we need the following lemma, see [2, page 145].

Lemma 3.2 Let $p$ be a prime number. Then $C_{p}$ does not contain any Latin subsquare of order $l, 1<l<p$.

In our example we present a product construction for which $m_{1} \neq m_{2}$ (using the notation preceding Theorem 2.1). For this we need a special biembedding of Latin squares. Let $C_{n}^{\varphi}$ be a Latin square of odd order $n \geq 5$ obtained from $C_{n}$ by the permutation of columns $\varphi=\left(\begin{array}{ccccccc}0 & 1 & 2 & 3 & 4 & \ldots & n-1 \\ 1 & n-1 & 0 & 2 & 3 & \ldots & n-2\end{array}\right)$. As an illustration, the square $C_{5}^{\varphi}$ is shown in Figure 2.

Lemma 3.3 If $n$ is odd, $n \geq 5$, then $C_{n} \bowtie C_{n}^{\varphi}$.

Proof. We check the rotations around all row, column and entry vertices. The rotation around row $i, 0 \leq i \leq n-1$, is

$$
r_{i}: c_{0} e_{i} c_{1} e_{1+i} c_{n-1} e_{n-1+i} c_{n-2} e_{n-2+i} \ldots c_{3} e_{3+i} c_{2} e_{2+i} .
$$

The rotations around columns are

$$
\begin{aligned}
c_{0} & : r_{0} e_{0} r_{n-2} e_{n-2} r_{n-4} e_{n-4} \ldots r_{3} e_{3} r_{1} e_{1} r_{n-1} e_{n-1} r_{n-3} e_{n-3} \ldots r_{2} e_{2}, \\
c_{1} & : r_{0} e_{1} r_{1} e_{2} r_{2} e_{3} \ldots r_{n-2} e_{n-1} r_{n-1} e_{0} \\
c_{i} & : r_{0} e_{i} r_{n-1} e_{n-1+i} r_{n-2} e_{n-2+i} \ldots r_{2} e_{2+i} r_{1} e_{1+i}, \\
c_{n-1} & : r_{0} e_{n-1} r_{n-2} e_{n-3} r_{n-4} e_{n-5} \ldots r_{3} e_{2} r_{1} e_{0} r_{n-1} e_{n-2} r_{n-3} e_{n-4} \ldots r_{2} e_{1},
\end{aligned}
$$

where $2 \leq i \leq n-2$. Observe that in the rotations around $c_{0}$ and $c_{n-1}$ we used the fact that $n$ is odd. The rotation around entry $i, 0 \leq i \leq n-1$, is

$$
e_{i} \quad: \quad r_{i} c_{0} r_{n-2+i} c_{2} r_{n-3+i} c_{3} r_{n-4+i} c_{4} \ldots r_{2+i} c_{n-2} r_{1+i} c_{n-1} r_{n-1+i} c_{1} .
$$

Thus, $C_{n} \bowtie C_{n}^{\varphi}$ as required.

We remark that the biembedding $C_{n} \bowtie C_{n}^{\varphi}$ can also be obtained by the voltage assignment construction presented in [6]. In the notation of [6], form $B(r, e ; \alpha)$, where 
$\alpha=(0,1, n-1, n-2, \ldots, 2)$. Then for $2 \leq i \leq n-2$ place vertex $c_{i}$ into the lifted image of the digon with voltages $i+1$ and $i$, and place $c_{0}, c_{1}, c_{n-1}$ respectively into the lifted images of the digons with voltages 2 and 0,0 and 1,1 and $n-1$.

In the following matrix $T$, in the first row we have the diagonal of $C_{n}$ and in the second row we have the diagonal of $C_{n}^{\varphi}, n$ being odd and $n \geq 5$.

$$
T=\left(\begin{array}{cccccccccccc}
0 & 2 & 4 & 6 & \ldots & n-1 & 1 & 3 & 5 & \ldots & n-2 \\
2 & 1 & 5 & 7 & \ldots & 0 & 2 & 4 & 6 & \ldots & 0
\end{array}\right)
$$

The highlighted entries determine a shared transversal $\mathcal{T}^{\varphi}$ containing $C_{n}(0,0), C_{n}^{\varphi}(1,1)$, $C_{n}(2,2), \quad \ldots, \quad C_{n}\left(\frac{n-1}{2}, \frac{n-1}{2}\right), \quad C_{n}^{\varphi}\left(\frac{n+1}{2}, \frac{n+1}{2}\right), \quad C_{n}\left(\frac{n+3}{2}, \frac{n+3}{2}\right), \ldots$, $C_{n}(n-1, n-1)$, so that $m_{1}=n-2$ and $m_{2}=2$.

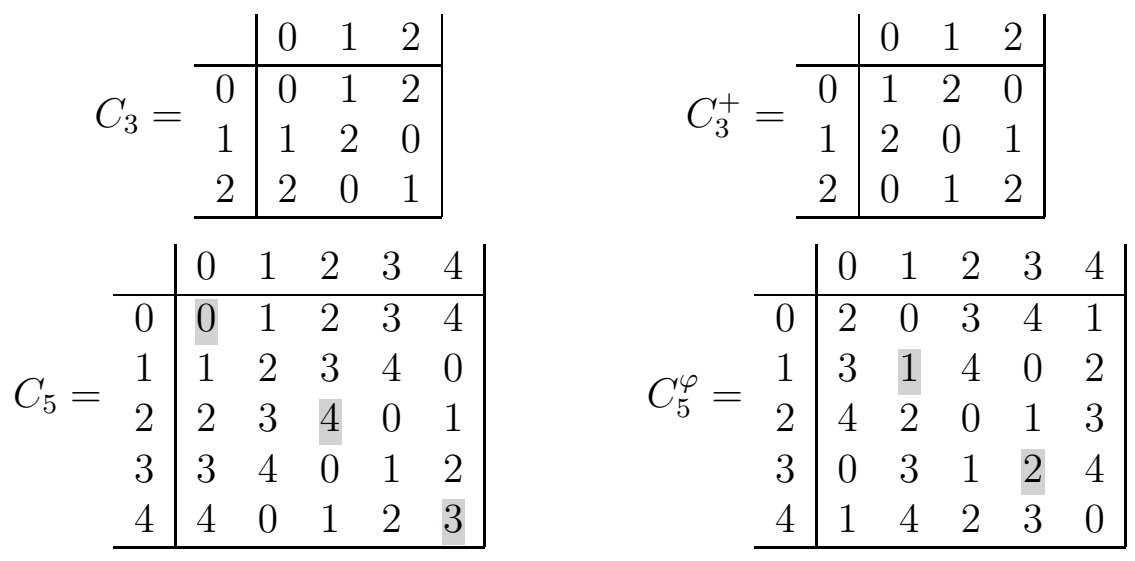

Figure 2: The Latin squares $C_{3}, C_{3}^{+}, C_{5}$ and $C_{5}^{\varphi}$.

Now we present our example. Denote by $C_{n}^{+}$the Latin square of order $n$ defined by $C_{n}^{+}(i, j)=i \oplus j \oplus 1,0 \leq i, j \leq n-1$, where the addition is modulo $n$. Then $C_{n} \bowtie C_{n}^{+}$, see for example $[5,6]$. Take $L=C_{n}, L^{\prime}=C_{n}^{+}, Q=C_{m}$ and $Q^{\prime}=C_{m}^{\varphi}, m$ being odd and $m \geq 5$. Then, by Corollary 2.2 , we have $C_{m}\left(C_{n}, \mathcal{T}^{\varphi}, C_{n}^{+}\right) \bowtie C_{m}^{\varphi}\left(C_{n}, \mathcal{T}^{\varphi}, C_{n}^{+}\right)$.

Figure 2 shows $C_{n}, C_{n}^{+}, C_{m}$ and $C_{m}^{\varphi}$ in the particular case when $n=3$ and $m=5$. The shared transversal $\mathcal{T}^{\varphi}$ is shown highlighted in $C_{5}$ and $C_{5}^{\varphi}$.

Put $D=C_{5}\left(C_{3}, \mathcal{T}^{\varphi}, C_{3}^{+}\right)$and $D^{\prime}=C_{5}^{\varphi}\left(C_{3}, \mathcal{T}^{\varphi}, C_{3}^{+}\right)$. The squares $D$ and $D^{\prime}$ are shown on Figure 3, where the entries corresponding to $\mathcal{T}^{\varphi}$ are highlighted. By Corollary 2.2 we have $D \bowtie D^{\prime}$.

Returning to the general case, we will show that if $p$ and $q$ are distinct odd primes, $q \geq 5$, then $\mu=C_{q}\left(C_{p}, \mathcal{T}^{\varphi}, C_{p}^{+}\right) \bowtie C_{q}^{\varphi}\left(C_{p}, \mathcal{T}^{\varphi}, C_{p}^{+}\right)$cannot be obtained in a non-trivial way by the construction presented in Theorem 1.1. Obviously, $\mu$ cannot be obtained in a non-trivial way by the construction presented in [3] because $C_{q}\left(C_{p}, \mathcal{T}^{\varphi}, C_{p}^{+}\right)$and $C_{q}^{\varphi}\left(C_{p}, \mathcal{T}^{\varphi}, C_{p}^{+}\right)$are Latin squares of odd order. 


\begin{tabular}{|c|c|c|c|c|c|c|c|c|c|c|c|c|c|c|c|c|}
\hline & & 0 & 1 & 2 & 3 & 4 & 5 & 6 & 7 & 8 & 9 & 10 & 11 & 12 & 13 & 14 \\
\hline & 0 & 1 & 2 & 0 & 3 & 4 & 5 & 6 & 7 & 8 & 9 & 10 & 11 & 12 & 13 & 14 \\
\hline & 1 & 2 & 0 & 1 & 4 & 5 & 3 & 7 & 8 & 6 & 10 & 11 & 9 & 13 & 14 & 12 \\
\hline & 2 & 0 & 1 & 2 & 5 & 3 & 4 & 8 & 6 & 7 & 11 & 9 & 10 & 14 & 12 & 13 \\
\hline & 3 & 3 & 4 & 5 & 6 & 7 & 8 & 9 & 10 & 11 & 12 & 13 & 14 & 0 & 1 & 2 \\
\hline & 4 & 4 & 5 & 3 & 7 & 8 & 6 & 10 & 11 & 9 & 13 & 14 & 12 & 1 & 2 & 0 \\
\hline & 5 & 5 & 3 & 4 & 8 & 6 & 7 & 11 & 9 & 10 & 14 & 12 & 13 & 2 & 0 & 1 \\
\hline & 6 & 6 & 7 & 8 & 9 & 10 & 11 & 13 & 14 & 12 & 0 & 1 & 2 & 3 & 4 & 5 \\
\hline & 7 & 7 & 8 & 6 & 10 & 11 & 9 & 14 & 12 & 13 & 1 & 2 & 0 & 4 & 5 & 3 \\
\hline & 8 & 8 & 6 & 7 & 11 & 9 & 10 & 12 & 13 & 14 & 2 & 0 & 1 & 5 & 3 & 4 \\
\hline & 9 & 9 & 10 & 11 & 12 & 13 & 14 & 0 & 1 & 2 & 3 & 4 & 5 & 6 & 7 & 8 \\
\hline & 10 & 10 & 11 & 9 & 13 & 14 & 12 & 1 & 2 & 0 & 4 & 5 & 3 & 7 & 8 & 6 \\
\hline & 11 & 11 & 9 & 10 & 14 & 12 & 13 & 2 & 0 & 1 & 5 & 3 & 4 & 8 & 6 & 7 \\
\hline & 12 & 12 & 13 & 14 & 0 & 1 & 2 & 3 & 4 & 5 & 6 & 7 & 8 & 10 & 11 & 9 \\
\hline & 13 & 13 & 14 & 12 & 1 & 2 & 0 & 4 & 5 & 3 & 7 & 8 & 6 & 11 & 9 & 10 \\
\hline & 14 & 14 & 12 & 13 & 2 & 0 & 1 & 5 & 3 & 4 & 8 & 6 & 7 & 9 & 10 & 11 \\
\hline & & 0 & 1 & 2 & 3 & 4 & 5 & 6 & 7 & 8 & 9 & 10 & 11 & 12 & 13 & 14 \\
\hline & 0 & 6 & 7 & 8 & 0 & 1 & 2 & 9 & 10 & 11 & 12 & 13 & 14 & 3 & 4 & 5 \\
\hline & 1 & 7 & 8 & 6 & 1 & 2 & 0 & 10 & 11 & 9 & 13 & 14 & 12 & 4 & 5 & 3 \\
\hline & 2 & 8 & 6 & 7 & 2 & 0 & 1 & 11 & 9 & 10 & 14 & 12 & 13 & 5 & 3 & 4 \\
\hline & 3 & 9 & 10 & 11 & 4 & 5 & 3 & 12 & 13 & 14 & 0 & 1 & 2 & 6 & 7 & 8 \\
\hline & 4 & 10 & 11 & 9 & 5 & 3 & 4 & 13 & 14 & 12 & 1 & 2 & 0 & 7 & 8 & 6 \\
\hline & 5 & 11 & 9 & 10 & 3 & 4 & 5 & 14 & 12 & 13 & 2 & 0 & 1 & 8 & 6 & 7 \\
\hline & 6 & 12 & 13 & 14 & 6 & 7 & 8 & 0 & 1 & 2 & 3 & 4 & 5 & 9 & 10 & 11 \\
\hline & 7 & 13 & 14 & 12 & 7 & 8 & 6 & 1 & 2 & 0 & 4 & 5 & 3 & 10 & 11 & 9 \\
\hline & 8 & 14 & 12 & 13 & 8 & 6 & 7 & 2 & 0 & 1 & 5 & 3 & 4 & 11 & 9 & 10 \\
\hline & 9 & 0 & 1 & 2 & 9 & 10 & 11 & 3 & 4 & 5 & 7 & 8 & 6 & 12 & 13 & 14 \\
\hline & 10 & 1 & 2 & 0 & 10 & 11 & 9 & 4 & 5 & 3 & 8 & 6 & 7 & 13 & 14 & 12 \\
\hline & 11 & 2 & 0 & 1 & 11 & 9 & 10 & 5 & 3 & 4 & 6 & 7 & 8 & 14 & 12 & 13 \\
\hline & 12 & 3 & 4 & 5 & 12 & 13 & 14 & 6 & 7 & 8 & 9 & 10 & 11 & 0 & 1 & 2 \\
\hline & 13 & 4 & 5 & 3 & 13 & 14 & 12 & 7 & 8 & 6 & 10 & 11 & 9 & 1 & 2 & 0 \\
\hline & 14 & 5 & 3 & 4 & 14 & 12 & 13 & 8 & 6 & 7 & 11 & 9 & 10 & 2 & 0 & 1 \\
\hline
\end{tabular}

Figure 3: The Latin squares $D=C_{5}\left(C_{3}, \mathcal{T}^{\varphi}, C_{3}^{+}\right)$and $D^{\prime}=C_{5}^{\varphi}\left(C_{3}, \mathcal{T}^{\varphi}, C_{3}^{+}\right)$.

Lemma 3.4 Let $p$ and $q$ be distinct odd primes, $q \geq 5$, and let $R \in$ $\left\{C_{q}\left(C_{p}, \mathcal{T}^{\varphi}, C_{p}^{+}\right), C_{q}^{\varphi}\left(C_{p}, \mathcal{T}^{\varphi}, C_{p}^{+}\right)\right\}$. Then $R$ is decomposable into Latin subsquares of order $l, 1<l<p q$, if and only if $l=p$. Moreover, such a decomposition is unique, formed by $q^{2}$ Latin squares $S_{u, v}, 0 \leq u, v \leq q-1$, defined by $S_{u, v}(i, j)=R(p u+i, p v+j)$ with entries $\left\{S_{u, v}(i, j) ; 0 \leq i, j \leq p-1\right\}=\{p w, p w+1, \ldots, p w+(p-1)\}$ for suitable $w$ depending on $u$ and $v$. 
Proof. If $R$ is decomposable into Latin subsquares of order $l, 1<l<p q$, then as $p$ and $q$ are distinct odd primes, we have either $l=p$ or $l=q$.

Fix $u$ and $v, 0 \leq u, v \leq q-1$, and denote by $S_{u, v}$ the sub-array of $R$ of order $p$, defined by $S_{u, v}(i, j)=R(p u+i, p v+j), 0 \leq i, j \leq p-1$. Put

$$
w_{u, v}= \begin{cases}C_{q}(u, v) & \text { if } R=C_{q}\left(C_{p}, \mathcal{T}^{\varphi}, C_{p}^{+}\right), \\ C_{q}^{\varphi}(u, v) & \text { if } R=C_{q}^{\varphi}\left(C_{p}, \mathcal{T}^{\varphi}, C_{p}^{+}\right) .\end{cases}
$$

Then for every $i$ and $j, 0 \leq i, j \leq p-1$, we have $S_{u, v}(i, j)=p w_{u, v}+k$, where $0 \leq k \leq p-1$, by Corollary 2.2. Since $S_{u, v}$ is a square sub-array of a Latin square and the order of $S_{u, v}$ is $p$, we have $\left\{S_{u, v}(i, j) ; 0 \leq i, j \leq p-1\right\}=\left\{p w_{u, v}, p w_{u, v}+1, \ldots, p w_{u, v}+(p-1)\right\}$ and $S_{u, v}$ is a Latin square. Obviously, $\left\{S_{u, v} ; 0 \leq u, v \leq q-1\right\}$ forms a decomposition of $R$. It only remains to show that this decomposition is unique.

As mentioned above, the entries of $S_{u, v}$ are $\left\{p w_{u, v}, p w_{u, v}+1, \ldots, p w_{u, v}+(p-1)\right\}$. Replace every subsquare $S_{u, v}$ of $R$ by a single cell with entry $w_{u, v}$ and denote the resulting square by $P$. Then $P=C_{q}$ if $R=C_{q}\left(C_{p}, \mathcal{T}^{\varphi}, C_{p}^{+}\right)$and $P=C_{q}^{\varphi}$ if $R=C_{q}^{\varphi}\left(C_{p}, \mathcal{T}^{\varphi}, C_{p}^{+}\right)$. Hence, $P$ is isotopic to a cyclic Latin square of order $q$.

Now suppose that $S$ is a Latin subsquare of $R$ of order $l$, such that $S$ contains cells from $S_{u_{1}, v_{1}}$ and $S_{u_{2}, v_{2}}$ for $\left(u_{1}, v_{1}\right) \neq\left(u_{2}, v_{2}\right)$. Consider a mapping $\rho$ from $S$ to $P$, such that the row $p u+i$, column $p v+j$ and entry $p w_{u, v}+k$ is mapped to row $u$, column $v$ and entry $w_{u, v}$, respectively. For fixed $u$ and $v$, all row, column, entry triples $(p u+i, p v+$ $\left.j, p w_{u, v}+k\right)$ are mapped to $\left(u, v, w_{u, v}\right)$ for any $i, j$ and $k, 0 \leq i, j, k \leq p-1$. Thus $\rho(S)$ is a Latin subsquare of $P$. By the assumption, $\rho(S)$ contains cells $\left(u_{1}, v_{1}\right)$ and $\left(u_{2}, v_{2}\right)$ for $\left(u_{1}, v_{1}\right) \neq\left(u_{2}, v_{2}\right)$, and so by Lemma $3.2 \rho(S)=P$. Thus, $S$ contains cells from every $S_{u, v}, 0 \leq u, v \leq q-1$. Suppose that $S$ contains $r$ cells from one row of $S_{0,0}$ with entries $p w_{0,0}+k_{1}, p w_{0,0}+k_{2}, \ldots, p w_{0,0}+k_{r}$. As the columns passing through these $r$ cells contain entries from the range $\left[p w_{0,0}, p w_{0,0}+(p-1)\right]$ only in $S_{0,0}$, the square $S$ contains $r^{2}$ cells from $S_{0,0}$. Consequently, all $S_{u, 0}, 0 \leq u \leq q-1$, contain $r^{2}$ cells of $S$, and hence all $S_{u, v}$, $0 \leq u, v \leq q-1$, contain $r^{2}$ cells of $S$. Thus, the order of $S$ is $r q$. Since $l \in\{p, q\}$ and $p$ and $q$ are distinct primes, we conclude that $r=1$ and $l=q$. It follows that $S$ contains rows $0+i_{0}, p+i_{1}, \ldots,(q-1) p+i_{q-1}$ and columns $0+j_{0}, p+j_{1}, \ldots,(q-1) p+j_{q-1}$, where $0 \leq i_{0}, j_{0}, \ldots, i_{q-1}, j_{q-1} \leq p-1$. Furthermore, for each $t, 0 \leq t \leq q-1, S$ contains exactly one entry from $[t p,(t+1) p-1]$.

To obtain a contradiction to the supposition at the start of the preceding paragraph we will consider the role of the shared transversal $\mathcal{T}^{\varphi}$. In order to do this we first make the following definition. Given a Latin square $L$ of order $n$ (and with arithmetic in $\mathbb{Z}_{n}$ ), we will call the set of cells $\{(0, j),(1, j-1), \ldots,(n-1, j-n+1)\}$ a back diagonal of $L$.

Firstly consider the case when $R=C_{q}\left(C_{p}, \mathcal{T}^{\varphi}, C_{p}^{+}\right)$. Subsquares $S_{u, v}$ are of two types. Type 1 subsquares are those for which $S_{u, v}(i, j)=p w_{u, v}+C_{p}(i, j)$, and type 2 are those for which $S_{u, v}(i, j)=p w_{u, v}+C_{p}^{+}(i, j)$. There are $q-2$ subsquares $S_{u, u}$ of type 2 , and all remaining subsquares $S_{u, v}$ are of type 1 . For cells of $S$ in type 1 subsquares we have $R\left(u p+i_{u}, v p+j_{v}\right)=p w_{u, v}+\left(i_{u} \oplus j_{v}\right)$, where $\oplus$ is considered in $\mathbb{Z}_{p}$. Consider the back diagonals of $S$. Since $m_{1}=q-2$, two of these back diagonals do not hit cells of type 2 subsquares. Assume that these diagonals contain cells $\left(i_{0}, g p+j_{g}\right)$ and $\left(i_{0},(g+h) p+j_{g+h}\right)$, 
$0 \leq g \leq q-1$ and $1 \leq h \leq q-1$. As $P$ is the cyclic square $C_{q}$, we have

$$
\begin{gathered}
i_{0} \oplus j_{g}=i_{1} \oplus j_{g-1}=i_{2} \oplus j_{g-2}=\ldots=i_{g-1} \oplus j_{g-q+1}, \\
i_{0} \oplus j_{g+h}=i_{1} \oplus j_{g+h-1}=i_{2} \oplus j_{g+h-2}=\ldots=i_{g-1} \oplus j_{g+h-q+1}
\end{gathered}
$$

Now subtracting $i_{0} \oplus j_{g}=i_{1} \oplus j_{g-1}$ from $i_{0} \oplus j_{g+h}=i_{1} \oplus j_{g+h-1}$ we obtain $j_{g+h} \ominus j_{g}=$ $j_{g+h-1} \ominus j_{g-1}$. Analogously we get

$$
\begin{aligned}
& j_{g+h} \ominus j_{g}=j_{g+h-1} \ominus j_{g-1}=\ldots=j_{g+h-q+1} \ominus j_{g-q+1} \text {, i.e., } \\
& j_{0+h} \ominus j_{0}=j_{1+h} \ominus j_{1}=\ldots=j_{q-1+h} \ominus j_{q-1} \text {. }
\end{aligned}
$$

As $1 \leq h \leq q-1$ and $q$ is prime, we have

$$
j_{h} \ominus j_{0}=j_{2 h} \ominus j_{h}=j_{3 h} \ominus j_{2 h}=\cdots=j_{(q-1) h} \ominus j_{(q-2) h}=j_{0} \ominus j_{(q-1) h} .
$$

Put $f=j_{h} \ominus j_{0}$. Then $f \in \mathbb{Z}_{p}$ and

$$
j_{h}=j_{0} \oplus f, \quad j_{2 h}=j_{0} \oplus 2 f, \quad \ldots, \quad j_{(q-1) h}=j_{0} \oplus(q-1) f, \quad j_{0}=j_{0} \oplus q f,
$$

the addition being in $\mathbb{Z}_{p}$. Since $p$ and $q$ are coprime and $0 \leq f \leq p-1$, we have $f=0$. Consequently $j_{0}=j_{1}=\cdots=j_{q-1}$ and analogously we can get $i_{0}=i_{1}=\cdots=i_{q-1}$. Recall that for each $t, 0 \leq t \leq q-1, S$ contains exactly one entry from $[t p,(t+1) p-1]$. For $S_{u, u}$ of type 2 we get $R\left(u p+i_{u}, u p+j_{u}\right)=p w_{u, u}+i_{u} \oplus j_{u} \oplus 1 \neq R\left((u-1) p+i_{u-1},(u+1) p+j_{u+1}\right)=$ $p w_{u, u}+i_{u} \oplus j_{u}$, so that $S$ is not a Latin subsquare of $R$.

Secondly consider the case when $R=C_{q}^{\varphi}\left(C_{p}, \mathcal{T}^{\varphi}, C_{p}^{+}\right)$. By reordering the columns of $R$ we can transform $R$ into a Latin square $R^{*}$ which has a similar structure to $C_{q}\left(C_{p}, \mathcal{T}^{\varphi}, C_{p}^{+}\right)$. Then $S_{u, v}^{*}$ and $P^{*}$ may be defined in relation to $R^{*}$ as $S_{u, v}$ and $P$ were defined in relation to $R$. As before, $P^{*}=C_{q}$. The only difference between the two cases is that because $m_{2}=2$, only two Latin subsquares $S_{u, v}^{*}$ of $R^{*}$ are of type 2, while all the others are of type 1. Obviously, there are two back diagonals in $S^{*}$ which do not hit the two type 2 subsquares. Thus, as in the previous case, it can be shown that $S^{*}$ is not a Latin subsquare of $R^{*}$, and consequently $S$ is not a Latin subsquare of $R$.

Hence, in either case, if $R$ is decomposable into Latin subsquares of order $l, 1<l<p q$, then every subsquare of the decomposition is a subsquare of $S_{u, v}$ for some $u$ and $v$, $0 \leq u, v \leq q-1$. Since $S_{u, v}$ is isotopic to the cyclic square $C_{p}$ and $p$ is a prime number, the squares of the decomposition are exactly $S_{u, v}, 0 \leq u, v \leq q-1$, by Lemma 3.2. That is, $R$ is decomposable into Latin subsquares of order $l$ if and only if $l=p$, and in such a case the decomposition is unique.

In the main theorem of this section we will utilize the decompositions of $C_{q}\left(C_{p}, \mathcal{T}^{\varphi}, C_{p}^{+}\right)$ and $C_{q}^{\varphi}\left(C_{p}, \mathcal{T}^{\varphi}, C_{p}^{+}\right)$derived in Lemma 3.4. Let $R$ be a Latin square of order $p q$, where $p$ and $q$ are distinct primes. Then $R$ will be called $p$-tidy if each $S_{u, v}, 0 \leq u, v \leq q-1$, defined by $S_{u, v}(i, j)=R(p u+i, p v+j), 0 \leq i, j \leq p-1$, is a Latin subsquare of $R$ with entries $\left\{S_{u, v}(i, j): 0 \leq i, j \leq p-1\right\}=\{p w, p w+1, \ldots, p w+(p-1)\}$ for some $w$ depending only on $u$ and $v$.

By Lemma 3.4, both $C_{q}\left(C_{p}, \mathcal{T}^{\varphi}, C_{p}^{+}\right)$and $C_{q}^{\varphi}\left(C_{p}, \mathcal{T}^{\varphi}, C_{p}^{+}\right)$are $p$-tidy Latin squares. But we have more. 
Lemma 3.5 Suppose that $R$ is a p-tidy Latin square. Let $R^{*}$ be obtained from $R$ by applying any one of the six possible permutations which mutually interchange the three partite sets of $R$, namely the rows, columns and entries. Then $R^{*}$ is a p-tidy Latin square.

Proof. First let $R^{\prime}$ be obtained from a $p$-tidy Latin square $R$ by interchanging the rows for columns and vice versa. Define $S_{v, u}^{\prime}, 0 \leq v, u \leq q-1$, square arrays of order $p$, such that $S_{v, u}^{\prime}(j, i)=R^{\prime}(p v+j, p u+i), 0 \leq j, i \leq p-1$. Then $S_{v, u}^{\prime}(j, i)=R(p u+i, p v+j)$. Since $R$ is a $p$-tidy Latin square, we have $\left\{S_{v, u}^{\prime}(i, j) ; 0 \leq i, j \leq p-1\right\}=\{R(p u+i, p v+j) ; 0 \leq$ $i, j \leq p-1\}=\{p w, p w+1, \ldots, p w+(p-1)\}$ for some $w$, so that $R^{\prime}$ is also a $p$-tidy Latin square.

Now let $R^{\prime}$ be obtained from a $p$-tidy Latin square $R$ by interchanging the rows for entries and vice versa. Then $R^{\prime}(p w+k, p v+j)=p u+i$ if and only if $R(p u+i, p v+j)=$ $p w+k, 0 \leq i, j, k \leq p-1$ and $0 \leq u, v, w \leq q-1$. Define $S_{w, v}^{\prime}$, square arrays of order $p$, such that $S_{w, v}^{\prime}(k, j)=R^{\prime}(p w+k, p v+j)$. Fix $w$ and $v$ and denote by $u_{w, v}$ a value such that $R\left(p u_{w, v}+i, p v\right)=p w$ for some $i, 0 \leq i \leq p-1$. Since $R$ is a $p$-tidy Latin square, we have $\left\{R\left(p u_{w, v}+i, p v+j\right) ; 0 \leq i, j \leq p-1\right\}=\{p w, p w+1, \ldots, p w+(p-1)\}$. Then $\left\{S_{w, v}^{\prime}(k, j) ; 0 \leq k, j \leq p-1\right\}=\left\{R^{\prime}(p w+k, p v+j) ; 0 \leq k, j \leq p-1\right\}=\left\{p u_{w, v}, p u_{w, v}+\right.$ $\left.1, \ldots, p u_{w, v}+(p-1)\right\}$, so that $R^{\prime}$ is also a $p$-tidy Latin square.

Since every one of the six possible permutations mutually interchanging the three partite sets of $R$ can be composed from the two involutions considered above, the square $R^{*}$ is a $p$-tidy Latin square.

We will say that a biembedding of Latin squares $\alpha=A \bowtie B$ is a $p$-tidy biembedding if both $A$ and $B$ are $p$-tidy Latin squares.

Theorem 3.6 Let $p$ and $q$ be distinct odd primes, $q \geq 5$, and let $\mu$ be the embedding $C_{q}\left(C_{p}, \mathcal{T}^{\varphi}, C_{p}^{+}\right) \bowtie C_{q}^{\varphi}\left(C_{p}, \mathcal{T}^{\varphi}, C_{p}^{+}\right)$. Then $\mu$ cannot be obtained by the product construction presented in Theorem 1.1 in a non-trivial way.

Proof. By way of contradiction, suppose that $\mu$ is obtained by Theorem 1.1 in a nontrivial way. Then there are $m(m>1)$ biembeddings $L \bowtie L_{u}^{\prime}, 0 \leq u \leq m-1$, of Latin squares of order $n$ and $n^{2}$ biembeddings $Q_{i, j} \bowtie Q_{i, j}^{\prime}, 0 \leq i, j \leq m-1$, of Latin squares of order $m$, all $Q_{i, j}^{\prime}$ sharing a common transversal $\mathcal{T}$, such that the embedding $\nu=A \bowtie A^{\prime}$ defined in Theorem 1.1 is isomorphic to $\mu$.

Suppose $\chi$ is an isomorphism from $\mu=C_{q}\left(C_{p}, \mathcal{T}^{\varphi}, C_{p}^{+}\right) \bowtie C_{q}^{\varphi}\left(C_{p}, \mathcal{T}^{\varphi}, C_{p}^{+}\right)$to $\nu=$ $A \bowtie A^{\prime}$. By Lemma 3.4, both the squares appearing in $\mu$ have a unique decomposition into non-trivial Latin subsquares, and these subsquares have order $p$. Hence $A$ and $A^{\prime}$ must also have such a decomposition. By Lemma 3.1, $A$ has a decomposition into Latin subsquares of side $m$ and, by assumption, $m>1$. Hence, $m=p$, and consequently $n=q$. The isomorphism $\chi$ must be of the form $\chi=\chi_{2} \chi_{1}$, where $\chi_{1}$ is one of the six possible permutations of the three sets $\{$ row labels $\},\{$ column labels $\}$, \{entries $\}$, and $\chi_{2}$ permutes the points within each of these sets. 
Now suppose that $\chi_{1} \mu=B_{1} \bowtie B_{1}^{\prime}=\mu^{\prime}$, say. Then $\chi_{2} \mu^{\prime}=\nu$, so that $\mu^{\prime}$ and $\nu$ are isomorphic under a mapping that only permutes points within each of the sets of row labels, column labels, and entries. By Lemma 3.5, $\mu^{\prime}$ is $p$-tidy. By Lemma 3.4, the unique decomposition of $B_{1}\left(B_{1}^{\prime}\right)$ into Latin subsquares of order $p$ is given by $\left\{S_{u, v}\right\}\left(\left\{S_{u, v}^{\prime}\right\}\right)$ where $0 \leq u, v \leq q-1$ and

$$
\begin{aligned}
& S_{u, v}(i, j)=B_{1}(p u+i, p v+j), 0 \leq i, j \leq p-1, \text { with entries } p w+k, 0 \leq k \leq p-1, \\
& S_{u, v}^{\prime}(i, j)=B_{1}^{\prime}(p u+i, p v+j), 0 \leq i, j \leq p-1, \text { with entries } p w+k, 0 \leq k \leq p-1 .
\end{aligned}
$$

The decomposition of $A$ into Latin subsquares of order $p$ is formed by $\left\{A_{u, v}\right\}$ where $0 \leq u, v \leq q-1$ and

$$
A_{u, v}(i, j)=A(q i+u, q j+v), 0 \leq i, j \leq p-1 \text {, with entries } q k+w, 0 \leq k \leq p-1 .
$$

Now define $\beta$, a permutation on $\{0,1, \ldots, p q-1\}$, by

$$
\beta(p u+i)=q i+u, 0 \leq i \leq p-1,0 \leq u \leq q-1 .
$$

Then define $\bar{\chi}$ on $\mu^{\prime}$ by applying $\beta$ to the row labels, to the column labels, and to the entries of $B_{1}$ and $B_{1}^{\prime}$ to form $\bar{\mu}$, say. Then $\bar{\mu}=\bar{\chi} \mu^{\prime}=\bar{\chi} \chi_{1} \mu$ and $\chi_{2} \bar{\chi}^{-1} \bar{\mu}=\chi_{2} \chi_{1} \mu=\chi \mu=\nu$. If $\bar{\mu}=B_{2} \bowtie B_{2}^{\prime}$, say, where $B_{2}=\bar{\chi}\left(B_{1}\right)$ and $B_{2}^{\prime}=\bar{\chi}\left(B_{1}^{\prime}\right)$, then the unique decomposition of $B_{2}\left(B_{2}^{\prime}\right)$ into Latin subsquares of order $p$, is given by

$$
\begin{aligned}
& \left.T_{u, v}(i, j)=B_{2}(q i+u, q j+v)\right), 0 \leq i, j \leq p-1, \text { with entries } q k+w, 0 \leq k \leq p-1, \\
& \left.T_{u, v}^{\prime}(i, j)=B_{2}^{\prime}(q i+u, q j+v)\right), 0 \leq i, j \leq p-1 \text {, with entries } q k+w, 0 \leq k \leq p-1 .
\end{aligned}
$$

The mapping $\chi_{2} \bar{\chi}^{-1}$ is a permutation of the points within each of the sets of row labels, column labels and entries. It takes $\bar{\mu}=B_{2} \bowtie B_{2}^{\prime}$ to $\nu=A \bowtie A^{\prime}$, in which the decomposition of $A$ is given by $\left\{A_{u, v}\right\}$. Since these decompositions are unique, each $A_{u, v}$ is the image of some $T_{u^{\prime}, v^{\prime}}$, or each $A_{u, v}$ is the image of some $T_{u^{\prime}, v^{\prime}}^{\prime}$. So, if $\chi_{2} \bar{\chi}^{-1}$ maps (say) the row points $q i_{1}+u, q i_{2}+u$ to $q i_{1}^{\prime}+u^{\prime}, q i_{2}^{\prime}+u^{\prime \prime}$, then $u^{\prime}=u^{\prime \prime}$, and likewise for column and entry points.

If we define $Z_{i, j}(u, v) \equiv T_{u, v}(i, j)(\bmod q)$, then

$$
Z_{i^{\prime}, j^{\prime}}(u, v) \equiv Z_{i, j}(u, v)(\bmod q), 0 \leq i, j, i^{\prime}, j^{\prime} \leq p-1,0 \leq u, v \leq q-1 .
$$

Thus, working modulo $q$, for fixed $u, v$ and varying $i, j$, the entries $T_{u, v}(i, j)$ are all equal and, by the argument in the previous paragraph, this remains the case after applying $\chi_{2} \bar{\chi}^{-1}$. So $B_{2}$ (and likewise $B_{2}^{\prime}$ ) may be decomposed into $p^{2}$ square sub-arrays of side $q$ (the $Z_{i, j}$ in the case of $B_{2}$ ) which are identical modulo $q$, by taking rows and columns $(i, j)$ modulo $p$, and this remains the case after applying $\chi_{2} \bar{\chi}^{-1}$.

Since either $\chi_{2} \bar{\chi}^{-1}\left(B_{2}\right)$ or $\chi_{2} \bar{\chi}^{-1}\left(B_{2}^{\prime}\right)$ is $A^{\prime}$, the same decomposition must apply to $A^{\prime}$. However,

$$
\begin{aligned}
A_{u, v}^{\prime}(i, j) & =A^{\prime}(q i+u, q j+v) \\
& =q Q_{u, v}^{\prime}(i, j)+\left\{\begin{array}{l}
L_{i}^{\prime}(u, v) \text { if }(i, j, k) \in \mathcal{T} \text { for some } k, \\
L(u, v) \text { otherwise. }
\end{array}\right. \\
& \equiv\left\{\begin{array}{l}
L_{i}^{\prime}(u, v) \text { if }(i, j, k) \in \mathcal{T} \text { for some } k, \\
L(u, v) \text { otherwise. }
\end{array}\right\}(\bmod q) .
\end{aligned}
$$


But then if $(i, j, k) \in \mathcal{T}$ and $\left(i^{\prime}, j^{\prime}, k^{\prime}\right) \notin \mathcal{T}$ are triples of $Q_{u, v}^{\prime}$, we have (modulo $q$ )

$$
A_{u, v}^{\prime}(i, j) \equiv L_{i}^{\prime}(u, v) \not \equiv L(u, v) \equiv A_{u, v}^{\prime}\left(i^{\prime}, j^{\prime}\right) .
$$

This is a contradiction. Hence $\mu$ and $\nu$ cannot be isomorphic.

Acknowledgement Part of this work commenced when the second author was visiting the first author and he thanks him for his hospitality. He also acknowledges partial support by Slovak research grants VEGA 1/0871/11 and APVV-0223-10.

\section{References}

[1] C. P. Bonnington, M. J. Grannell, T. S. Griggs and J. Širáň, Exponential families of nonisomorphic triangulations of complete graphs, J. Combin. Theory Ser. B 78 (2000), 169-184.

[2] C. J. Colbourn and J. H. Dinitz (editors), The CRC Handbook of Combinatorial Designs, 2nd Edition, CRC Press, Boca Raton, 2006.

[3] D. M. Donovan, A. Drápal, M. J. Grannell, T. S. Griggs and J. G. Lefevre, Quarterregular biembeddings of Latin squares, Discrete Math. 310 (2010), 692-699.

[4] M. J. Grannell and T. S. Griggs, A lower bound for the number of triangular embeddings of some complete graphs and complete regular tripartite graphs, J. Combin. Theory Ser. B, 98 (2008) 637-650.

[5] M. J. Grannell, T. S. Griggs and M. Knor, Biembeddings of Latin squares and Hamiltonian decompositions, Glasgow Math. J. 46 (2004), 443-457.

[6] M. J. Grannell, T. S. Griggs, M. Knor and J. Širáň, Triangulations of orientable surfaces by complete tripartite graphs, Discrete Math. 306 (2006), 600-606.

[7] M. J. Grannell, T. S. Griggs and M. Knor, On biembeddings of Latin squares, Electron. J. Comb. 16 (2009), 12pp.

[8] M. J. Grannell, T. S. Griggs and J. Širáň, Recursive constructions for triangulations, J. Graph Theory 39 (2002), 87-107.

[9] M. J. Grannell and M. Knor, A lower bound for the number of orientable triangular embeddings of some complete graphs, J. Combin. Theory Ser. B 100 (2010) 216-225.

[10] J. L. Gross and T. W. Tucker, Topological Graph Theory, John Wiley, New York (1987).

[11] V. P. Korzhik, Exponentially many nonisomorphic orientable triangular embeddings of $K_{12 s}$, Discrete Math. 308 (2008), 1046-1071.

[12] V. P. Korzhik, Exponentially many nonisomorphic orientable triangular embeddings of $K_{12 s+3}$, Discrete Math. 309 (2009), 852-866.

[13] V. P. Korzhik and H.-J. Voss, On the number of nonisomorphic orientable regular embeddings of complete graphs, J. Combin. Theory Ser. B 81 (2001), 58-76. 
[14] V. P. Korzhik and H.-J. Voss, Exponential families of nonisomorphic non-triangular orientable genus embeddings of complete graphs, J. Combin. Theory Ser. B 86 (2002), 186-211.

[15] V. P. Korzhik and H.-J. Voss, Exponential families of nonisomorphic nonorientable genus embeddings of complete graphs, J. Combin. Theory Ser. B 91 (2004), 253-287.

[16] S. Lawrencenko, S. Negami and A. T. White, Three nonisomorphic triangulations of an orientable surface with the same complete graph, Discrete Math. 135 (1994), 367-369.

[17] G. Ringel, Map Color Theorem, Springer-Verlag, New York, 1974. 